\title{
Hypoxia affects mesenchymal stromal cell osteogenic differentiation and angiogenic factor expression
}

\author{
Authors: E. Potier ${ }^{1}$, E. Ferreira ${ }^{1}$, R. Andriamanalijaona ${ }^{2}$, J. P. Pujol ${ }^{2}$, K. Oudina ${ }^{1}$, D. Logeart-Avramoglou ${ }^{1}$, H. Petite $^{1}$ \\ Affiliations: \\ 1 Laboratoire de Recherches Orthopédiques (B2OA), UMR CNRS 7052, Université Denis Diderot - Paris VII, Paris, France \\ 2 Laboratory of Connective Tissue Biochemistry, Faculty of Medicine, Caen, France
}

Key words: Mesenchymal stromal cells; Hypoxia; Osteogenic differentiation; Angiogenic factor; Cell survival

To cite this article: Potier E, Ferreira E, Andriamanalijaona R, Pujol JP, Oudina K, Logeart-Avramoglou D, Petite H (2007). Hypoxia affects mesenchymal stromal cell osteogenic differentiation and angiogenic factor expression. Bone, 40(4): $1078-87$. doi: 10.1016/j.bone.2006.11.024

Document Version: Accepted manuscript including changes made at the peer-review stage

\begin{abstract}
Mesenchymal stromal cells (MSCs) seeded onto biocompatible scaffolds have been proposed for repairing bone defects. When transplanted in vivo, MSCs (expanded in vitro in $21 \% \mathrm{O}_{2}$ ) undergo temporary oxygen deprivation due to the lack of pre-existing blood vessels within these scaffolds. In the present study, the effects of temporary (48-hour) exposure to hypoxia $\left(\leq 1 \% \mathrm{O}_{2}\right)$ on primary human MSC survival and osteogenic potential were investigated. Temporary exposure of MSCs to hypoxia had no effect on MSC survival, but resulted in (i) persistent (up to 14 days post exposure) down-regulation of cbfa-1/Runx2, osteocalcin and type I collagen and (ii) permanent (up to 28 days post exposure) up-regulation of osteopontin mRNA expressions. Since angiogenesis is known to contribute crucially to alleviating hypoxia, the effects of temporary hypoxia on angiogenic factor expression by MSCs were also assessed. Temporary hypoxia led to a 2-fold increase in VEGF expression at both the mRNA and protein levels. Other growth factors and cytokines secreted by MSCs under control conditions (namely bFGF, TGF $\beta 1$ and IL-8) were not affected by temporary exposure to hypoxia. All in all, these results indicate that temporary exposure of MSCs to hypoxia leads to limited stimulation of angiogenic factor secretion but to persistent down-regulation of several osteoblastic markers, which suggests that exposure of MSCs transplanted in vivo to hypoxia may affect their bone forming potential. These findings prompt for the development of appropriate cell culture or in vivo transplantation conditions preserving the full osteogenic potential of MSCs.
\end{abstract}

\section{Introduction}

Mesenchymal stromal cells (MSCs) loaded onto biocompatible scaffolds have been proposed for restoring function of lost or injured connective tissue, including bone $(6,33,38)$. Physiological oxygen tensions in bone are about $12.5 \% \mathrm{O} 2(22)$ but fall to $1 \%$ $\mathrm{O} 2$ in fracture hematoma $(4,22)$. In tissue engineering applications, implanted MSCs undergo temporary oxygen deprivation, which may be considered as similar to fracture hematoma (i.e., $1 \% \mathrm{O} 2$ ) due to the disruption of the host vascular system (as the result of injury and/or surgery) and the lack of preexisting vascular networks within these scaffolds.

These drastic conditions of transplantation can lead to the death or functional impairment of MSCs, which can affect their ultimate bone forming potential. The exact effects of hypoxia on osteoprogenitor or osteoblast-like cells have not been clearly established, however, as several studies demonstrated a negative impact on cell growth $(37,46)$ and differentiation $(37,42,48)$, whereas others have shown that hypoxia has positive effects on cell proliferation (48) and osteoblastic differentiation (51). These discrepancies may be due to the differences between the cell types (primary $(42,48,51)$ and cell lines $(37,42,46))$, species (rat $(48$, $51)$, human $(37,42)$ and mouse $(42,46))$ and hypoxic conditions (from $0.02 \%$ to $5 \% \mathrm{O} 2$ ) used. Since the success of bone reconstruction methods based on the use of engineered constructs depends on the maintenance of viable and functional MSCs, it is of particular interest to elucidate the effects of temporary hypoxia on primary human MSC survival and osteogenic potential.

MSCs secrete a wide variety of angiogenic factors (including vascular endothelial growth factor (VEGF) (27), transforming growth factor $\beta 1$ (TGF $\beta 1)(21,43)$, and basic fibroblast growth factor (bFGF) $(21,27)$ ) and may therefore modulate angiogenic processes and participate in the vascular invasion of engineered contructs. Since effective neo-vascularization is crucial for shortening the hypoxic episodes to which transplanted MSCs are exposed, it seemed to be worth investigating the stimulatory effects of hypoxia on angiogenic factor expression by MSCs.

The aim of the present study was therefore to investigate the effects of temporary hypoxia on primary human MSC (hMSC) proliferation, osteogenic potential and angiogenic factor expression. In this study, $\mathrm{O}_{2}$ tensions $\leq 4 \%$ are termed hypoxic conditions (as these conditions represent the hypoxia to which 
hMSCs transplanted in vivo are subjected) and $21 \% \mathrm{O}_{2}$ tensions are termed control conditions (as these conditions represent standard cell culture conditions). Cell viability was assessed after exposing hMSCs to hypoxic conditions during various periods of time. Osteogenic differentiation was assessed after temporary (48-hour) exposure of hMSCs to either control or hypoxic conditions followed by different periods of osteogenic cell culture. Expression of several angiogenic factors by hMSCs involved in new blood vessel formation (VEGF, bFGF and TGF $\beta$ ) and maturation (platelet derived growth factor BB (PDGF-BB)) was assessed after temporary (48-hour) exposure of hMSCs to either control or hypoxic conditions.

\section{Materials and Methods}

\section{Hypoxia}

Hypoxia was obtained using a sealed jar (Oxoid Ltd, Basingstoke, United Kingdom) containing an oxygen chelator (AnaeroGen, Oxoid Ltd) (18). Twice a day, the $\mathrm{pO}_{2}$ was measured diving an oxygen electrode directly into cell culture medium $(\mathrm{pH}$ : 7.2) and using an Oxylab $\mathrm{pO}_{2} \mathrm{TM}$ (Oxford Optronix; Oxford, United Kingdom). The hypoxic system was left closed throughout the period of experimentation.

\section{Cell culture}

Human mesenchymal stromal cells (hMSCs) were isolated from tibia bone marrow specimens obtained as discarded tissue during routine bone surgery (spinal fusion) in keeping with local regulations. Bone marrows were obtained from 3 donors ( 2 males and 1 female; 14-16 years old). hMSCs were isolated using a procedure previously described in the literature $(16,39)$. Briefly, cells were harvested by gently flushing bone marrow samples with alpha Minimum Essential Medium ( $\alpha$ MEM, Sigma) containing $10 \%$ fetal bovine serum (FBS, PAA Laboratories) and 1\% antibiotic and anti-mycotic solution (PAA Laboratories). When the hMSCs reached $60-70 \%$ confluence, they were detached and cryopreserved at P1 (90\% FBS, $10 \%$ DMSO). For each experiment, a new batch of hMSCs was thawed and cultured. Cells from each donor were cultured separately. Human endothelial cells (EC, kindly provided by Dr Le Ricousse-Roussanne) were cultured in Medium 199 (Sigma) containing 20\% FBS supplemented with $15 \mathrm{mM}$ HEPES (Sigma) and $10 \mathrm{ng} / \mathrm{ml}$ rhVEGF165 (R\&D Systems) (31).

\section{Multipotency of hMSCs}

Induction of osteogenic differentiation. hMSCs (passage P7) were cultured in osteogenic medium consisting of aMEM containing $10 \% \mathrm{FBS}, 10^{-7} \mathrm{M}$ dexamethasone, $0.15 \mathrm{mM}$ ascorbate2-phosphate (Sigma), and $2 \mathrm{mM} \beta$-glycerophosphate (Sigma) (10). After 10 and 20 days of culture, the cells were fixed in PBS containing 1\% PFA and stained with a NBT/TCIP kit (Molecular Probes) to evaluate the alkaline phosphatase (ALP) activity. Calcium deposition was assayed by using the Von-Kossa staining method (5). After 10 and 20 days of culture, mRNA extraction, cDNA synthesis and RT-PCR were performed as described in the "RT-PCR assays" section to assess the transcription levels of osteogenic markers (osteocalcin and osterix).

Induction of chondrogenic differentiation. hMSCs (passage P7; $2 \times 10^{5}$ cells) suspended in $0.5 \mathrm{ml}$ of chondrogenic medium were centrifuged for $2 \mathrm{~min}$ at $500 \mathrm{~g}$. The chondrogenic medium used contained $\alpha$ MEM supplemented with $6.25 \mu \mathrm{g} / \mathrm{ml}$ insulin, 6.26 $\mu \mathrm{g} / \mathrm{ml}$ transferrin (Sigma), $6.25 \mu \mathrm{g} / \mathrm{ml}$ selenious acid (Sigma), 5.35 $\mu \mathrm{g} / \mathrm{ml}$ linoleic acid (Sigma), $1.25 \mu \mathrm{g} / \mathrm{ml}$ bovine serum albumin (Sigma), $1 \mathrm{mM}$ pyruvate (Sigma), and $37.5 \mathrm{ng} / \mathrm{ml}$ ascorbate-2phosphate (10). After centrifugation, pellets of hMSCs were cultured in chondrogenic medium supplemented with $10 \mathrm{ng} / \mathrm{ml}$ TGFß1 (R\&D Systems) and $10^{-7} \mathrm{M}$ dexamethasone (10). After 20 and 30 days of cell culture, hMSC pellets were cryo-preserved ($80^{\circ} \mathrm{C}$ ) until immuno-histological analysis to detect the presence of human type II collagen. Human type II collagen protein was detected using a goat polyclonal IgG anti-human type II collagen antibody (200 $\mu \mathrm{g} / \mathrm{ml}$; Santa Cruz Biotechnology). Peroxidaseconjugated anti-goat IgG antibody (1:200; Vectastain ABC kit; Vector) was used as the secondary antibody. Peroxidase activity was monitored using a Vectastain ABC kit. Sections were counterstained using haematoxylin.

Induction of adipogenic differentiation. hMSCs (passage P7) were cultured in adipogenic medium consisting of $\alpha \mathrm{MEM}$ containing 10\% FBS, $5 \mu \mathrm{g} / \mathrm{ml}$ insulin (Boehringer Manheim), $10^{-7}$ $\mathrm{M}$ dexamethasone (Sigma), $0.5 \mathrm{mM}$ isobutylmethylxanthine (Sigma), and $60 \mu \mathrm{M}$ indomethacin (Sigma) (10). After 10 and 20 days of culture, the cells were fixed in PBS containing $1 \%$ paraformaldehyde (PFA, Sigma) and stained with Oil Red O (Sigma) (11). After 10 and 20 days of cell culture, mRNA extraction, cDNA synthesis and RT-PCR were performed as described in the "RT-PCR assays" section to assess the transcription levels of adipogenic markers (fatty acid binding protein $4(\mathrm{aP} 2)$ and peroxisome proliferator-activated receptor $(\operatorname{PPAR} \gamma))$.

\section{Cell death assays}

hMSCs (passage P5) were plated at 5,000 cells $/ \mathrm{cm}^{2}$ and allowed to adhere overnight. Cells were subsequently exposed to hypoxic conditions (without medium change) for different periods of time. Cell death was assessed by image analysis (Leica Qwin software) after staining with the Live/Dead viability/cytotoxicity kit (Molecular Probes).

hMSC osteogenic differentiation after exposure to temporary hypoxia

hMSCs (passage P5) were plated at 5,000 cells $/ \mathrm{cm}^{2}$ and allowed to adhere overnight. After exposure of hMSCs either to hypoxic or control conditions for 48 hours, the cell culture supernatant medium was replaced by osteogenic medium and hMSCs were cultured in control conditions for 0,14 and 28 days. mRNA extraction, cDNA synthesis and RT-PCR were then performed as described in the "RT-PCR assays" section to assess the transcription levels of osteogenic markers (osteocalcin, ALP, type I collagen, osteopontin, bone sialoprotein (BSP), core binding factor alpha sub-unit 1 (cbfa-1/Runx2) and bone morphogenetic protein-2 (BMP-2)).

\section{RT-PCR assays}

Cytoplasmic mRNA was extracted from cell layers using an RNeasy mini kit (Qiagen) and digested with RNase-free DNase (Qiagen) in line with the manufacturer's instructions. cDNA synthesis was performed using a Thermoscript kit (Invitrogen) and Oligo DT primers $(50 \mu \mathrm{M})$. PCRs were performed on an iCycler using a Multiplex PCR kit (Qiagen) with $15 \mathrm{ng}$ of cDNA and 0.2 $\mu \mathrm{M}$ of each of the primers (for primer sequences see Table 1 , supplemental data). After a 10 -min denaturation step at $95^{\circ} \mathrm{C}$, cDNA was amplified in PCR cycles consisting of a three-step PCR: a 30-sec denaturation step at $95^{\circ} \mathrm{C}$, a 90 -sec annealing step at $60^{\circ} \mathrm{C}$, and a 90 -sec elongation step at $72^{\circ} \mathrm{C}$. An additional $10-$ min elongation cycle was conducted at $72^{\circ} \mathrm{C}$. PCR products were 
analyzed by performing agarose gel electrophoresis and ethidium bromide staining. In each PCR, ribosomal protein L13a (RPL13a) was used as the endogenous reference gene (for primer sequences see Table 1). RPL13a was chosen among the 5 housekeeping genes tested (RPL13a, ßactin, glyceraldehyde-3-phosphate dehydrogenase, $18 \mathrm{~S}$ ribosomal RNA, and hypoxanthine phosphoribosyltransferase 1) as the most "stable" housekeeping gene in hMSCs exposed to hypoxic conditions. cDNA from ECs was used as the positive control in the angiogenic growth factor mRNA expression assays. Semi-quantitation of the PCR products was performed using Quantity One software (BioRad). Expression of target genes was normalized taking the respective RPL13a expression levels.

\section{Real Time PCR assays}

mRNA extraction and reverse transcription were conducted as described in the "RT-PCR assays" section. Real Time PCR assays were performed on the ABI Prism 7000 SDS (Applied Biosystems) using the SYBR Green Mastermix Plus (Eurogentec) with $1.5 \mathrm{ng}$ of cDNA (1/50 diluted) and 400-600 nM of each of the primers (for primer sequences see Table 2, supplemental data). After a 10min denaturation step at $95^{\circ} \mathrm{C}$, cDNA was amplified by performing two-step PCR cycles: a 15 -sec step at $95^{\circ} \mathrm{C}$, followed by a 1 -min step at $60^{\circ} \mathrm{C}$. In each Real Time PCR assay, one of the cDNA used was diluted $(1 / 2 ; 1 / 4 ; 1 / 8)$ in order to establish a standard curve and define the exact number of cycles corresponding to $100 \%$ efficiency of polymerization. Reactions were performed in triplicate and expression of target genes was normalized taking the respective RPL13a expression levels. Relative quantities of cDNA were calculated from the number of cycles corresponding to $100 \%$ efficiency of polymerization, using the $2^{- \text {DeltaDeltaCT }}$ method (32).

\section{ELISA assays}

After exposing hMSCs to either hypoxic or control conditions for 48 hours, the supernatant media were collected, centrifuged at $13,000 \mathrm{~g}$ at $4^{\circ} \mathrm{C}$ for $10 \mathrm{~min}$, collected, and kept at $-80^{\circ} \mathrm{C}$ until ELISA assays were performed. VEGF, bFGF, and Interleukin-8 (IL-8) expressions were assayed using ELISA kits from R\&D Systems (Quantikine) in line with the manufacturer's instructions. TGF $\beta 1$ expression was assayed using an ELISA assay developed at our laboratory (34), after activating TGF $\beta 1$ by acidifying the cell culture supernatant media (50).

\section{Angiogenesis antibody array assays}

The levels of expression of 20 growth factors and cytokines were determined using the RayBio® human angiogenesis antibody array (Ray Biotech, Inc, Norcross, GA, USA). After exposing hMSCs to either hypoxic or control conditions for 48 hours, the supernatant media were collected and stored as described in the "ELISA assays" section. Protein-antibody complexes were revealed by chemoluminescence in line with the manufacturer's instructions and the results were photographed on Xomat AM film (Kodak). The following growth factors and cytokines were detected by the RayBio® angiogenesis antibody arrays: angiogenin, RANTES, leptin, thrombopoeitin, epidermal growth factor, epithelial neutrophil-activating protein 78, bFGF, growth regulated oncogene, interferon $\gamma$, VEGF, VEGF-D, insulin-like growth factor-1, interleukin 6, interleukin 8, monocyte chemoattractant protein 1 (MCP-1), PDGF, placenta growth factor, TGF $\beta 1$, tissue inhibitors of metalloproteinases 1 (TIMP-1), and tissue inhibitors of metalloproteinases-2 (TIMP-2).

\section{Statistical analysis}

Data are expressed as means \pm standard deviations. Statistical analysis was performed using an ANOVA with a Fisher post hoc test. The results were taken to be significant at a probability level of $\mathrm{P}<0.05$.

\section{Results}

\section{Multipotency of hMSCs}

In order to determine the multipotency of the human mesenchymal stromal cells (hMSCs) used in this study, hMSCs were cultured in either osteogenic, chondrogenic, or adipogenic differentiation medium.

Culture of hMSCs in osteogenic medium for 10 and 20 days increased the levels of alkaline phosphatase (ALP) activity (Fig.1A). Osteogenic differentiation of hMSCs was confirmed by the expression of the osteogenic differentiation markers osterix and osteocalcin (Fig.1A).

Culture of hMSCs in chondrogenic medium for 30 days resulted in the expression of the type II collagen (marker of the chondrogenic differentiation) in the cell cytoplasm and extracellular matrix (Fig.1B). Control sections incubated with secondary antibody alone showed negative staining patterns (Fig.1B).

Culture of hMSCs in adipogenic medium for 20 days resulted in the development of several clusters of adipocytes containing intracellular lipid vacuoles, which stained positive with Oil Red O (Fig.1C). Expression of fatty acid binding protein 4 (aP2) and peroxisome proliferator activated receptor $\gamma$ (PPAR $\gamma$ ) (markers of the adipogenic differentiation) by hMSCs (Fig.1C) confirmed the ability of these cells to differentiate along the adipogenic lineage.

All these results confirm that the hMSCs used in this study are multipotent cells, since they are capable of differentiating along the osteogenic, adipogenic and chondrogenic lineages as previously demonstrated by numerous studies (for review: $(3,26,30,40)$ ). But, even when hMSCs were committed to the osteoblastic lineage, the extracellular matrix did not mineralize after 30 days of cell culture in osteogenic medium. These results suggest that the culture conditions used in this study were suboptimal to preserve full biological function of hMSCs.

\section{Hypoxic model}

In order to check the validity of the model for hypoxia used in this study, the $\mathrm{pO}_{2}$ levels were monitored in the sealed jar during 5 days and without exposing to atmospheric oxygen tensions. Moderate hypoxic conditions $\left(\mathrm{pO}_{2}=4 \% \mathrm{O}_{2}\right)$ may be said to have been reached within 24 hours. Severe hypoxic conditions $\left(\mathrm{pO}_{2}<\right.$ $\left.1 \% \mathrm{O}_{2}\right)$ may be considered as reached after 48 hours. The $\mathrm{pO}_{2}$ levels in the cell culture medium gradually decreased, reaching a plateau corresponding to values of around $0.25 \% \mathrm{O}_{2}$ after 72 hours (Fig.2).

\section{Effects of prolonged hypoxia on hMSC survival}

To investigate the effects of hypoxia on cell survival, hMSCs were exposed to hypoxic conditions for 48, 72 and 120 hours. Exposure of hMSCs to prolonged (120 hours) hypoxic conditions resulted in limited rates of cell death (Fig.3; $35.5 \pm 18.5 \%$ ), whereas temporary hypoxia did not affect hMSC survival. 


\section{Effects of temporary hypoxia on the osteogenic potential of $h M S C s$}

Having established that temporary hypoxia has no effect on hMSC survival, its effects on hMSC osteogenic potential were assessed. After 48-hour exposure to hypoxic or control conditions, hMSCs were transferred to osteogenic medium and osteogenic differentiation was assessed by performing RT-PCR assays to detect the expression of several osteogenic markers. The levels of cbfa-1/Runx2, osteocalcin and type I collagen expression were checked by performing quantitative real-time PCR assays.

Similar levels of ALP, bone morphogenetic protein 2 (BMP2) and bone sialoprotein (BSP) expression were observed in hMSCs exposed to either hypoxic or control conditions at all time periods of osteogenic culture tested (Fig.4).

Osteopontin expression increased after exposure of hMSCs to hypoxic conditions at all osteogenic culture times tested ( 0 days: 2.6-fold; 14 days: 12 -fold; 28 days: 8 -fold) (Fig.4).

The levels of expression of cbfa-1/Runx 2 and osteocalcin were slightly down-regulated after 0 and 14 days of osteogenic culture by temporary exposure to hypoxic conditions ( 0.5 -fold with cbfa1/Runx2; 0.7-fold with osteocalcin), as assessed by quantitative real time PCR assays (Fig.5). After 28 days of osteogenic culture, however, the levels of cbfa-1/Runx 2 and osteocalcin expressed by hMSCs exposed to hypoxic conditions were similar to those exposed to control conditions.

Type I collagen expression was permanently down-regulated after 48-hour exposure of hMSCs to hypoxic conditions (approximately 0.4-fold at all the osteogenic culture times tested), but this decrease was statistically significant only on days 0 and 28 of osteogenic culture (Fig.5).

Effects of temporary hypoxia on the mRNA expression of angiogenic factors by hMSCs

Effects of temporary hypoxia on angiogenic factor expression by hMSCs were investigated. mRNA expression of angiogenic factors was assessed by performing RT-PCR assays after exposing hMSCs to either hypoxic or control conditions for 48 hours. Expression levels of key angiogenic factors (namely vascular endothelial growth factor (VEGF), basic fibroblast growth factor (bFGF), transforming growth factor $\beta 1, \beta 2$ and $\beta 3$ (TGF- $\beta 1$, TGF$\beta 2$, and TGF- $\beta 3$ )) and those of VEGF receptor 1 and receptor 2 were studied.

No expression of PDGF-BB, VEGF receptor 1 or VEGF receptor 2 was detected under any of the conditions tested with hMSCs. However, the RT-PCR conditions used were suitable for the detection of PDGF-BB, VEGF receptor 1 and VEGF receptor 2 , as these factors were detected with endothelial cells (EC) (data not shown).

Similar levels of TGF $\beta 1$ and TGF $\beta 2$ expression were detected after exposing hMSCs to either hypoxic or control conditions for 48 hours (Fig.6). The levels of TGF $\beta 3$ expression decreased after exposure to hypoxic conditions for 48 hours (TGF $33 /$ RPL13a ratio: $0.21 \pm 0.05)$, in comparison with TGF $\beta 3$ expression obtained under control conditions $(0.89 \pm 0.8)$ (Fig.6).

Conversely, expression levels of bFGF and VEGF increased when hMSCs were exposed to hypoxic conditions for 48 hours (bFGF/RPL13a ratio: $0.71 \pm 0.13$; VEGF/RPL13a ratio: $1.51 \pm$ $0.05)$, in comparison to results obtained under control conditions $(0.14 \pm 0.01$ and $0.25 \pm 0.24$ respectively) ( $\underline{\text { Fig.6 }}$.
Effects of temporary hypoxia on the protein secretion levels of three major regulators of angiogenesis by hMSCs

Since the secretion of angiogenic factors is required to induce angiogenesis, the levels of protein secretion of three major regulators of angiogenesis (namely VEGF, TGF $\beta 1$, and bFGF which were previously detected at the mRNA level) were assessed by performing ELISA assays after exposing hMSCs to either hypoxic or control conditions for 48 hours.

To measure the TGF $\beta 1$ content of the cell culture supernatant media, acid activation of samples was required. Without this activation, no TGF $\beta 1$ secretion was detectable (data not shown). TGF $\beta 1$ secretion by hMSCs exposed to hypoxic conditions $(270 \pm$ $70 \mathrm{pg} / \mathrm{ml}$ ) was down-regulated in comparison with TGF $\beta 1$ secretion obtained under control conditions $(570 \pm 270 \mathrm{pg} / \mathrm{ml})$, but did not reach statistical significance (Fig.7A).

bFGF secretion decreased, but not significantly, in response to exposure of hMSCs to hypoxic conditions $(0.4 \pm 0.3 \mathrm{pg} / \mathrm{ml})$ in comparison with control conditions $(1.2 \pm 0.5 \mathrm{pg} / \mathrm{ml})$ (Fig.7B). Even under control conditions, however, hMSCs were found to secrete small quantities of bFGF.

Contrary to what occurred with TGF $\beta 1$ and bFGF, VEGF secretion by hMSCs exposed to hypoxic conditions (1640 \pm 260 $\mathrm{pg} / \mathrm{ml}$ ) increased 2-fold in comparison with the results obtained under control conditions $(880 \pm 100 \mathrm{pg} / \mathrm{ml})$ (Fig.7C).

Neither TGF $\beta 1$, bFGF nor VEGF were detected in control medium alone (data not shown).

Effects of temporary hypoxia on the protein secretion of various growth factors and cytokines by hMSCs

To further investigate the effects of temporary and moderate hypoxia on hMSCs, the secretion levels of various growth factors and cytokines involved in angiogenic processes were monitored using angiogenesis antibody arrays after exposing hMSCs to either hypoxic or control conditions for 48 hours. Any changes in the growth factor and cytokine secretion levels were checked by performing conventional ELISA assays.

Similar levels of secretion of Interleukin-6 (IL-6), Monocyte Chemoattractant Protein-1 (MCP-1), Tissue inhibitor MetalloProteinases 1 and 2 (TIMP-1 and TIMP-2) were observed in hMSCs, whether they were exposed to hypoxic or control conditions.

Interleukin-8 (IL-8) secretion was up-regulated in two out of the three donors tested by exposing hMSCs to hypoxic conditions. These results were confirmed by the results of ELISA assays, which showed that IL-8 secretion by hMSCs exposed to hypoxic conditions increased $(780 \pm 390 \mathrm{pg} / \mathrm{ml})$ in comparison to what occurred under control conditions $(440 \pm 230 \mathrm{pg} / \mathrm{ml})$. This upregulation was not statistically significant, however, due to the great variability existing between donors.

Other growth factors and cytokines tested using angiogenesis antibody arrays were not detected in hMSCs exposed to control or hypoxic conditions (data not shown). Neither cytokines nor growth factors were detected by angiogenesis antibody arrays incubated in control medium alone (data not shown).

\section{Discussion}

The first step in the present study consisted in evaluating the effects of reduced oxygen tensions on hMSC survival. Our results showed that 120-hour exposure to hypoxia resulted in increased cell death rates, when 48- or 72-hour exposure did not, but those 
cell death rates may have been underestimated as the method used in the present study did not consider floatting dead cells. The mechanisms underlying hMSC death upon oxygen deprivation are unclear at present. A previous study conducted on rat MSCs, however, offers some clues as it reported the induction of caspase dependent apoptosis under brief ( 24 hours) oxygen and serum deprivation (nuclear shrinkage, chromation condensation, decrease in cell size, and loss of menbrane integrity) (54). The hMSC viability does not seem to be affected by short-term ( $<72$-hour) hypoxia which are in agreement with previously published data $(42,49)$. Grayson et al. reported that long-term culture of hMSCs under hypoxic conditions $\left(2 \% \mathrm{O}_{2}\right)$ resulted in decreased cell proliferation but not in increased apoptosis after 9, 16 or 24 days of cell culture (17). These findings, combined with our own, suggest that hypoxia leads only to moderate cell death and that the surviving hMSCs are still able to proliferate. The ultimate bone forming ability of engineered constructs relies, however, on the survival of "functional" hMSCs. The second step in the present study was therefore to assess the effects of temporary hypoxia on hMSC osteogenic potential by drawing up transcriptional profiles of osteoblast membraneous and extra-cellular matrix molecules (ALP, osteocalcin, osteopontin and type I collagen), those of a growth factor stimulating osteoblast differentiation (BMP2) and those of a transcription factor regulating bone formation (cbfa1/Runx2).

Our results show that a slight down-regulation of cbfa-1/Runx2 expression occurs after temporary exposure to hypoxia, persisting for 14 days after the end of the hypoxic episode. Cbfa-1/Runx 2 transcription factor plays an essential role in controlling osteoblastic differentiation (for a review: $(13,29)$ ) and its inhibition is associated with a large decrease in the rate of bone formation (14). Similar long-lasting inhibition of osteocalcin, a late osteogenic differentiation marker, confirmed the inhibition of osteoblastic maturation of hMSCs resulting from temporary exposure to hypoxia. As occurred with type I collagen, its level of expression was durably and strongly inhibited by temporary exposure to hypoxia. Type I collagen is the main component of bone matrix and plays a central role in the mineralization process. Long-term inhibition of cbfa-1/Runx2, osteocalcin and type I collagen expressions strongly suggest that temporary exposure to hypoxia may inhibit the osteoblastic differentiation of hMSCs. Literature conducted on other cell types (human $(35,37)$ and rat (48) osteoblasts) reports that their osteogenic differentiation is impaired by temporary exposure to hypoxia (decreased ALP activity, collagen type I, osteocalcin and cbfa-1/Runx2 expressions). Conversely, Salim et al reported that exposure of hMSCs to hypoxic $\left(2 \% \mathrm{O}_{2}\right)$ conditions did not affect their terminal differentiation (42). The discrepancies observed between this study and our results may be explained by different time of exposure to hypoxic conditions ( 24 hours and 48 hours respectively), suggesting that hMSCs are able to face hypoxia for a short period of time ( $<48$ hours) without losing their osteogenic potential.

Surprisingly, neither the expression of BSP, which is regulated by cbfa-1/Runx2 at both mRNA (14) and protein levels (23), nor that of ALP, the enzymatic activity of which has been previously reported to be down-regulated under hypoxic conditions (37, 48, 49 ), were found here to be affected by temporary exposure to hypoxia. In the case of BSP expression, the down-regulation of cbfa-1/Runx2 observed in the present study may be too weak to significantly inhibit BSP expression. Moreover, Park et al. (37) have reported that the inhibitory effect of hypoxia on the osteoblastic differentiation of a human osteosarcoma cell line is time-dependent: the longer the hypoxic exposure time, the higher the down-regulation of osteoblastic marker expression. These results suggest that exposure times longer than those used in the present study (48-hour) may nonetheless induce a down-regulation of mRNA expression of BSP or ALP.

Osteopontin expression by hMSCs was permanently increased, on the contrary, by temporary exposure to hypoxia. Up-regulation of osteopontin induced by hypoxia has been previously observed in many other cell types, including mouse osteocytes (19), rat aortic vascular smooth muscle cells (45), and human renal proximal tubular epithelial cells (20). In bone, osteopontin mediates the attachment of several cell types, including osteoblasts, endothelial cells and osteoclasts (for a review: (9)). This molecule plays an important role in bone remodelling and osteoclast recruitment processes, as its absence (in knock-out mice) led to impaired bone loss after ovariectomy (53) and decreased resorption of subcutaneously implanted bone discs (2). As far as the effects of its up-regulation are concerned, however, the results of previous studies are confusing as positive effects on rat osteoblast maturation (28) as well as negative effects on osteoblastic differentiation of the MC3T3 cell line (24) have been reported. But the most striking property of osteopontin may be its ability to promote macrophage infiltration (for a review: (9)). Increased osteopontin expression by transplanted hMSCs may therefore culminate in attracting macrophages to the bone defect site and exacerbating the inflammatory process. The exact effects of increased osteopontin expression on bone formation by hMSCs, i.e., whether it stimulates bone formation processes or attracts osteoclasts and macrophages to bone defect site, still remain to be determined.

Angiogenesis, a crucial process for oxygen supply to cells, is modulated by several proangiogenic factors (for a review: $(7,47)$ ), which expression is stimulated by HIF-1 (hypoxia inducible factor 1), a transcription factor activated by hypoxia (for review see: :(41, 52)). The third step in the present study was therefore to assess the effects of temporary exposure to hypoxia on angiogenic factor expression by hMSCs. Our results showed that a 2-fold upregulation of VEGF expression by hMSCs occurs under hypoxic conditions at both mRNA and protein levels. These findings are in agreement with previous reports that hypoxia increases VEGF expression in the MC3T3 cell line (46). Expression of other growth factors and cytokines studied here, although regulated at the mRNA level, were not affected at the protein level by temporary exposure to hypoxia. The bFGF expression, indeed, was upregulated by exposure to hypoxia at the mRNA but not at the protein levels. The discrepancies between mRNA and protein may be explained by shorter half-life of bFGF, lower translation efficiency or the absence of post-translational modification under hypoxia. Moreover, several studies comparing genomic and proteomic analyses report moderate or no correlation between RNA and protein expression $(8,25)$.

Even so, MSCs are able to durably enhance (for up to 28 days) tissue reperfusion when transplanted into ischemic myocardium $(15,44)$. Stimulation of VEGF alone does not suffice, however, to trigger the formation of functional vascular networks, as attempts to accelerate vascularization by over-expressing VEGF (using a genetic system) resulted in the formation of immature, leaky blood vessels in mice $(1,12,36)$. These findings suggest either that the secretion levels of multiple angiogenic factors by MSCs, even if they are not up-regulated by hypoxia, suffice to promote vascular invasion of ischemic tissues; that MSCs secrete other growth 
factors and cytokines involved in angiogenesis, the expression levels of which have not been studied here; or that MSCs may indirectely promote angiogenesis in vivo by stimulating the secretion of angiogenic factors by other cell types.

The present study shows that exposure of primary hMSCs to temporary hypoxia results in persistent down-regulation of cbfa1/Runx2, osteocalcin and type I collagen levels, but in the upregulation of osteopontin expression, which may therefore limit in vivo bone forming potential of hMSCs. This study, however, only addressed the effects of a transient 48-hour exposure to hypoxia with osteogenic differentiation conducted in hyperoxic conditions $\left(21 \% \mathrm{O}_{2}\right)$. When transplanted in vivo, MSCs undergo temporary oxygen deprivation but will never come back to hyperoxic conditions as the maximum oxygen tensions reported either in blood (22) or in diaphyseal bone (4) do not exceed $12.5 \% \mathrm{O}_{2}$. One may then expect more disastrous effects on hMSC osteoblastic differentiation when cells are transplanted in vivo than when they are exposed to in vitro 48-hour hypoxia. It may be therefore of great interest to determine what in vitro hMSC culture conditions are most appropriate for preserving their osteogenic potential after their in vivo implantation.

\section{Acknowledgments}

We thank Dr. Michele Guerre-Millo for providing the sealed jar for hypoxic cell culture conditions, Dr. Sylviane Dennler and Dr. Alain Mauviel for their expert assistance with the RT-PCR assays, and Dr. Sophie Le Ricousse-Roussanne for providing endothelial cells. We would also like to express special thanks to Professor Christophe Glorion and Dr. Jean-Sebastien Sylvestre for their help.

\section{Disclosure Statement}

The authors declare no competing financial interests.

\section{References}

1. Ash, J. D., and Overbeek, P. A. Lens-specific VEGF-A expression induces angioblast migration and proliferation and stimulates angiogenic remodeling. Dev Biol 223:383-98; 2000.

2. Asou, Y., Rittling, S. R., Yoshitake, H., Tsuji, K., Shinomiya, K., Nifuji, A., Denhardt, D. T., and Noda, M. Osteopontin facilitates angiogenesis, accumulation of osteoclasts, and resorption in ectopic bone. Endocrinology 142:1325-32; 2001.

3. Barry, F. P., and Murphy, J. M. Mesenchymal stem cells: clinical applications and biological characterization. The International Journal of Biochemistry and Cell Biology:568-84.; 2004.

4. Brighton, C. T., and Krebs, A. G. Oxygen tension of healing fractures in the rabbit. J Bone Joint Surg Am 54:323-32; 1972.

5. Bruder, S. P., Jaiswal, N., and Haynesworth, S. E. Growth kinetics, self-renewal, and the osteogenic potential of purified human mesenchymal stem cells during extensive subcultivation and following cryopreservation. J Cell Biochem 64:278-94.; 1997.

6. Cancedda, R., Bianchi, G., Derubeis, A., and Quarto, R. Cell therapy for bone disease: a review of current status. Stem Cells 21:610-9; 2003.

7. Carmeliet, P. Angiogenesis in health and disease. Nat Med 9:653-60; 2003.

8. Chen, G., Gharib, T. G., Huang, C.-C., Taylor, J. M. G., Misek, D. E., Kardia, S. L. R., Giordano, T. J., Iannettoni, M. D., Orringer, M. B., Hanash, S. M., and Beer, D. G. Discordant Protein and mRNA Expression in Lung Adenocarcinomas. Mol Cell Proteomics:304-13; 2002.

9. Denhardt, D. T., Giachelli, C. M., and Rittling, S. R. Role of osteopontin in cellular signaling and toxicant injury. Annu Rev Pharmacol Toxicol 41:723-49; 2001.
10. Dennis, J. E., Carbillet, J. P., Caplan, A. I., and Charbord, P. The STRO-1+ marrow cell population is multipotential. Cells Tissues Organs 170:73-82; 2002.

11. Diascro, D. D., Jr., Vogel, R. L., Johnson, T. E., Witherup, K. M., Pitzenberger, S. M., Rutledge, S. J., Prescott, D. J., Rodan, G. A., and Schmidt, A. High fatty acid content in rabbit serum is responsible for the differentiation of osteoblasts into adipocyte-like cells. J Bone Miner Res 13:96-106; 1998

12. Dor, Y., Djonov, V., Abramovitch, R., Itin, A., Fishman, G. I., Carmeliet, P., Goelman, G., and Keshet, E. Conditional switching of VEGF provides new insights into adult neovascularization and pro-angiogenic therapy. Embo J 21:1939-47; 2002.

13. Ducy, P. Cbfa1: a molecular switch in osteoblast biology. Dev Dyn 219:461-71; 2000.

14. Ducy, P., Starbuck, M., Priemel, M., Shen, J., Pinero, G., Geoffroy, V., Amling, M., and Karsenty, G. A Cbfa1-dependent genetic pathway controls bone formation beyond embryonic development. Genes Dev 13:1025-36; 1999.

15. Fazel, S., Chen, L., Weisel, R. D., Angoulvant, D., Seneviratne, C., Fazel, A., Cheung, P., Lam, J., Fedak, P. W., Yau, T. M., and Li, R. K. Cell transplantation preserves cardiac function after infarction by infarct stabilization: augmentation by stem cell factor. J Thorac Cardiovasc Surg 130:1310; 2005

16. Friedenstein, A. J., Chailakhjan, R. K., and Lalykina, K. S. The development of fibroblast colonies in monolayer cultures of guinea- pig bone marrow and spleen cells. Cell Tissue Kinet 3:393-403.; 1970.

17. Grayson, W. L., Zhao, F., Izadpanah, R., Bunnell, B., and Ma, T. Effects of hypoxia on human mesenchymal stem cell expansion and plasticity in 3D constructs. J Cell Physiol 207:331-9; 2006.

18. Grosfeld, A., Turban, S., Andre, J., Cauzac, M., Challier, J. C., Hauguel-de Mouzon, S., and Guerre-Millo, M. Transcriptional effect of hypoxia on placental leptin. FEBS Lett 502:122-6; 2001.

19. Gross, T. S., King, K. A., Rabaia, N. A., Pathare, P., and Srinivasan, S. Upregulation of osteopontin by osteocytes deprived of mechanical loading or oxygen. J Bone Miner Res 20:250-6; 2005.

20. Hampel, D. J., Sansome, C., Romanov, V. I., Kowalski, A. J., Denhardt, D. T., and Goligorsky, M. S. Osteopontin traffic in hypoxic renal epithelial cells. Nephron Exp Nephrol 94:e66-76; 2003.

21. Han, S. K., Yoon, T. H., Lee, D. G., Lee, M. A., and Kim, W. K. Potential of human bone marrow stromal cells to accelerate wound healing in vitro. Ann Plast Surg 55:414-9; 2005.

22. Heppenstall, R. B., Grislis, G., and Hunt, T. K. Tissue gas tensions and oxygen consumption in healing bone defects. Clin Orthop Relat Res:357-65; 1975

23. Hoshi, K., Komori, T., and Ozawa, H. Morphological characterization of skeletal cells in Cbfa1-deficient mice. Bone 25:639-51; 1999.

24. Huang, W., Carlsen, B., Rudkin, G., Berry, M., Ishida, K., Yamaguchi, D. T., and Miller, T. A. Osteopontin is a negative regulator of proliferation and differentiation in MC3T3-E1 pre-osteoblastic cells. Bone 34:799-808; 2004.

25. Huber, M., Bahr, I., Krätzschmar, J. R., Becker, A., Müller, E.C., Donner, P., Pohlenz, H.-D., Schneider, M. R., and Sommer, A. Comparison of Proteomic and Genomic Analyses of the Human Breast Cancer Cell Line T47D and the Antiestrogen-resistant Derivative T47D_r*. Mol Cell Proteomics:43-55; 2004.

26. Jorgensen, C., Gordeladze, J., and Noel, D. Tissue engineering through autologous mesenchymal stem cells. Curr Opin Biotechnol 15:406$10 ; 2004$.

27. Kinnaird, T., Stabile, E., Burnett, M. S., Shou, M., Lee, C. W., Barr, S., Fuchs, S., and Epstein, S. E. Local delivery of marrow-derived stromal cells augments collateral perfusion through paracrine mechanisms. Circulation 109:1543-9; 2004.

28. Kojima, H., Uede, T., and Uemura, T. In vitro and in vivo effects of the overexpression of osteopontin on osteoblast differentiation using a recombinant adenoviral vector. J Biochem (Tokyo) 136:377-86; 2004

29. Komori, T. Regulation of skeletal development by the Runx family of transcription factors. J Cell Biochem 95:445-53; 2005. 
30. Krampera, M., Pizzolo, G., Aprili, G., and Franchini Mesenchymal stem cells for bone, cartilage, tendon and skeletal muscle repair. Bone; 2006.

31. Le Ricousse-Roussanne, S., Barateau, V., Contreres, J. O., Boval, B., Kraus-Berthier, L., and Tobelem, G. Ex vivo differentiated endothelial and smooth muscle cells from human cord blood progenitors home to the angiogenic tumor vasculature. Cardiovasc Res 62:176-84; 2004.

32. Livak, K. J., and Schmittgen, T. D. Analysis of relative gene expression data using real-time quantitative PCR and the 2(-Delta Delta C(T)) Method. Methods 25:402-8; 2001.

33. Logeart-Avramoglou, D., Anagnostou, F., Bizios, R., and Petite, H. Engineering bone: challenges and obstacles. J Cell Mol Med 9:72-84; 2005.

34. Maire, M., Logeart-Avramoglou, D., Degat, M. C., and Chaubet, F. Retention of transforming growth factor beta1 using functionalized dextran-based hydrogels. Biomaterials 26:1771-80; 2005.

35. Matsuda, N., Morita, N., Matsuda, K., and Watanabe, M. Proliferation and Differentiation of Human Osteoblastic Cells Associated with Differential Activation of MAP Kinases in Response to Epidermal Growth Factor, Hypoxia, and Mechanical Stress in Vitro. Biochem Biophys Res Commun:350-4; 1998.

36. Ozawa, C. R., Banfi, A., Glazer, N. L., Thurston, G., Springer, M. L., Kraft, P. E., McDonald, D. M., and Blau, H. M. Microenvironmental VEGF concentration, not total dose, determines a threshold between normal and aberrant angiogenesis. J Clin Invest 113:516-27; 2004.

37. Park, J. H., Park, B. H., Kim, H. K., Park, T. S., and Baek, H. S. Hypoxia decreases Runx2/Cbfa1 expression in human osteoblast-like cells. Mol Cell Endocrinol 192:197-203; 2002.

38. Petite, H., Viateau, V., Bensaid, W., Meunier, A., de Pollak, C., Bourguignon, M., Oudina, K., Sedel, L., and Guillemin, G. Tissueengineered bone regeneration. Nat Biotechnol 18:959-63.; 2000.

39. Pittenger, M. F., Mackay, A. M., Beck, S. C., Jaiswal, R. K., Douglas, R., Mosca, J. D., Moorman, M. A., Simonetti, D. W., Craig, S., and Marshak, D. R. Multilineage potential of adult human mesenchymal stem cells. Science 284:143-7.; 1999.

40. Prockop, D. J. Marrow stromal cells as stem cells for nonhematopoietic tissues. Science 276:71-4.; 1997.

41. Pugh, C. W., and Ratcliffe, P. J. Regulation of angiogenesis by hypoxia: role of the HIF system. Nat Med 9:677-84; 2003.

42. Salim, A., Nacamuli, R. P., Morgan, E. F., Giaccia, A. J., and Longaker, M. T. Transient changes in oxygen tension inhibit osteogenic differentiation and Runx2 expression in osteoblasts. J Biol Chem 279:40007-16; 2004.

43. Sensebe, L., Mortensen, B. T., Fixe, P., Herve, P., and Charbord, P. Cytokines active on granulomonopoiesis: release and consumption by human marrow myoid [corrected] stromal cells. $\mathrm{Br} \mathrm{J}$ Haematol 98:274-82; 1997.

44. Shyu, K. G., Wang, B. W., Hung, H. F., Chang, C. C., and Shih, D. T. Mesenchymal stem cells are superior to angiogenic growth factor genes for improving myocardial performance in the mouse model of acute myocardial infarction. J Biomed Sci 13:47-58; 2006.

45. Sodhi, C. P., Phadke, S. A., Batlle, D., and Sahai, A. Hypoxia stimulates osteopontin expression and proliferation of cultured vascular smooth muscle cells: potentiation by high glucose. Diabetes 50:1482-90; 2001.

46. Steinbrech, D. S., Mehrara, B. J., Saadeh, P. B., Chin, G., Dudziak, M. E., Gerrets, R. P., Gittes, G. K., and Longaker, M. T. Hypoxia regulates VEGF expression and cellular proliferation by osteoblasts in vitro. Plast Reconstr Surg 104:738-47; 1999.

47. Tonini, T., Rossi, F., and Claudio, P. P. Molecular basis of angiogenesis and cancer. Oncogene 22:6549-56; 2003.

48. Tuncay, O. C., Ho, D., and Barker, M. K. Oxygen tension regulates osteoblast function. Am J Orthod Dentofacial Orthop 105:457-63; 1994.

49. Utting, J. C., Robins, S. P., Brandao-Burch, A., Orriss, I. R., Behar, J., and Arnett, T. R. Hypoxia inhibits the growth, differentiation and bone-forming capacity of rat osteoblasts. Exp Cell Res; 2006.

50. van Waarde, M. A., van Assen, A. J., Kampinga, H. H., Konings, A. W., and Vujaskovic, Z. Quantification of transforming growth factor-beta in biological material using cells transfected with a plasminogen activator inhibitor-1 promoter-luciferase construct. Anal Biochem 247:45$51 ; 1997$.

51. Warren, S. M., Steinbrech, D. S., Mehrara, B. J., Saadeh, P. B., Greenwald, J. A., Spector, J. A., Bouletreau, P. J., and Longaker, M. T. Hypoxia regulates osteoblast gene expression. J Surg Res 99:147-55; 2001.

52. Wenger, R. H. Cellular adaptation to hypoxia: O2-sensing protein hydroxylases, hypoxia-inducible transcription factors, and $\mathrm{O} 2-$ regulated gene expression. Faseb J 16:1151-62; 2002.

53. Yoshitake, H., Rittling, S. R., Denhardt, D. T., and Noda, M. Osteopontin-deficient mice are resistant to ovariectomy-induced bone resorption. Proc Natl Acad Sci U S A 96:8156-60; 1999.

54. Zhu, W., Chen, J., Cong, X., Hu, S., and Chen, X. Hypoxia and serum deprivation-induced apoptosis in mesenchymal stem cells. Stem Cells 24:416-25; 2006.

Corresponding author:

Hervé Petite

B2OA, UMR CNRS 7052

Faculté de Médecine Lariboisière-Saint-Louis

10 Avenue de Verdun

75010 , Paris, France

Tel. +33 (0)1-44-89-78-21

Fax. $+33(0) 1-44-89-78-22$

Email : herve.petite@univ-paris-diderot.fr 
Table 1. Primer sequences for target and housekeeping genes used in RT-PCR assays.

* The accession number is the GenBank ${ }^{\mathrm{TM}}$ accession number. aP2: fatty acid binding protein 4; PPAR $\square$ : peroxisome proliferator activated receptor gamma; GAPDH: glyceraldehyde-3-phosphate dehydrogenase; VEGF: vascular endothelial growth factor; TGF $\square$ : transforming growth factor beta; bFGF: basic fibroblast growth factor; PDGF-BB: platelet derived growth factor - BB; VEGF-R1: VEGF receptor 1 (Flt-1); VEGF-R2: VEGF receptor 2 (KDR); Type I coll.: type I collagen; cbfa-1/Runx2: core binding factor alpha 1 subunit/Runx2; BMP2: bone morphogenetic protein 2; BSP: bone sialoprotein; ALP: alkaline phosphatase; RPL13a: ribosomal protein L13a.

\begin{tabular}{|c|c|c|c|c|}
\hline Gene & Accession gene * & Sequence 5'-3' & $\begin{array}{l}\text { Number of } \\
\text { PCR cycles }\end{array}$ & $\begin{array}{l}\text { Size of PCR } \\
\text { product (bp) }\end{array}$ \\
\hline \multirow{2}{*}{ aP2 } & \multirow{2}{*}{ NM_001442 } & TCAGTGTGAATGGGGATGTGA & \multirow{2}{*}{27} & \multirow{2}{*}{253} \\
\hline & & CTCGTGGAAGTGACGCCTTT & & \\
\hline \multirow{2}{*}{ PPARg } & \multirow{2}{*}{ NM_015869 } & GACCACTCCCATTCCTTTGA & \multirow{2}{*}{32} & \multirow{2}{*}{752} \\
\hline & & ACCTCTTTGCTCTGCTCCTG & & \\
\hline \multirow{2}{*}{ Osterix } & \multirow{2}{*}{ AF477981 } & CTGAAGAATGGGTGGGGAAG & \multirow{2}{*}{32} & \multirow{2}{*}{284} \\
\hline & & TGAGACAGCAGGGGACAGAA & & \\
\hline \multirow{2}{*}{ Osteocalcin } & \multirow{2}{*}{ NM_199173 } & CTCACACTCCTCGCCCTATT & \multirow{2}{*}{32} & \multirow{2}{*}{165} \\
\hline & & TCCCAGCCATTGATACAGGT & & \\
\hline \multirow{2}{*}{ GAPDH } & \multirow{2}{*}{ M33197 } & CGGATTTGGTCGTATTGGGC & \multirow{2}{*}{20} & \multirow{2}{*}{920} \\
\hline & & GTCATACCAGGAAATGAGCTTG & & \\
\hline \multirow{2}{*}{ VEGF } & \multirow{2}{*}{ NM_003376 } & GCACCCATGGCAGAAGGAGG & \multirow{2}{*}{24} & \multirow{2}{*}{263} \\
\hline & & CCTTGGTGAGGTTTGATCCGCATA & & \\
\hline \multirow{2}{*}{ TGFb1 } & \multirow{2}{*}{ NM_000660.1 } & GGTCACCCGCGTGCTAATGG & 24 & 546 \\
\hline & & GTGGAGCTGAAGCAATAGTTGG & & \\
\hline TGFb? & NM 003238 & CCGCCCACTTTCTACAGACCC & 33 & 184 \\
\hline 1GFD2 & NM_005238 & GCGCTGGGTGGGAGATGTTAA & 33 & 184 \\
\hline TGFb3 & NM 003239 & CATAAATTCGACATGATCCAGGG & 30 & 386 \\
\hline 1GFDS & & GGCTGAAAGGTGTGACATGG & & \\
\hline bFGF & NM 002006 & GGAGAAGAGCGACCCTCACATCAAG & 24 & 234 \\
\hline DFGF & NM_002000 & CCAGTTCGTTTCAGTGCCACATACCAA & 24 & 234 \\
\hline PDGF-BB & NM 033016 & TTTCTCACCTGGACAGGTCG & 33 & 226 \\
\hline PDGF-BB & NM_053010 & AAAGAGTGGACCTGTCCAGC & 33 & 220 \\
\hline VEGF-R1 & $\mathrm{AF} 063657$ & CGACCTTGGTTGTGGCTGACT & 33 & 654 \\
\hline & & CCCTTCTGGTTGGTGGCTTTG & & \\
\hline VEGF-R2 & $\mathrm{AF} 063658$ & AACAAAGTCGGGAGAGGA & 33 & 750 \\
\hline VEGT-R2 & АГО050ग0 & TGACAAGAAGTAGCCAGAAGA & & 100 \\
\hline ALP & NM 000478 & TACAACACCAATGCCCAGGT & 30 & 696 \\
\hline & & TTCCACCAGCAAGAAGAAGC & & \\
\hline BMP2 & NM 001200 & CCAGAAACGAGTGGGAAAAC & 33 & 615 \\
\hline Dintz & NMIOUIZOO & GTGGCAGTAAAAGGCGTGAT & 35 & 015 \\
\hline BSP & NM 004967 & AGAAAGCGAAGCAGAAGTGG & 33 & 435 \\
\hline & & TAGCCATCGTAGCCTTGTCC & & \\
\hline Osteonontin & X13694 & GCAACCGAAGTTTTCACTCC & 33 & 339 \\
\hline Usteopontm & Х15094 & GCTCTCATCATTGGCTTTCC & 35 & 359 \\
\hline chfa-1/Runx2 & NM 004348 & CCTCTGACTTCTGCCTCTGG & 30 & 295 \\
\hline & & TATGGAGTGCTGCTGGTCTG & & \\
\hline Tyne I Coll. & NM 000088 & CTCCCCAGAAGACACAGGAA & 21 & 632 \\
\hline & & ACAAGTCCCCATCCACAAAA & 21 & \\
\hline RPL13a & NM 012423 & GGTCCTGGTGCTTGATGGT & 21 & 854 \\
\hline & & TTCTGGCTCCCTTTCACTCA & & \\
\hline
\end{tabular}


Table 2. Primer sequences for target and housekeeping genes used in real time PCR assays.

* The accession number is the GenBank ${ }^{\mathrm{TM}}$ accession number. cbfa-1/Runx2: core binding factor alpha 1 subunit/Runx2; Type I coll.: type I collagen; RPL13a: ribosomal protein L13a.

\begin{tabular}{lllc} 
Gene & Accession gene & Sequence 5'-3' & $\begin{array}{c}\text { Size of PCR } \\
\text { product (bp) }\end{array}$ \\
\hline Osteocalcin & NM_199173 & $\begin{array}{l}\text { CGGTGCAGAGTCCAGCAAA } \\
\text { GGTAGCGCCTGGGTCTCTTC }\end{array}$ & 80 \\
\hline cbfa-1/Runx2 & NM_004348 & $\begin{array}{l}\text { GTACAGCTTTAAGGATTCCCTCAATTC } \\
\text { TTGCTAATGCTTCGTGTTTCCA }\end{array}$ & 85 \\
\hline Type I Coll. & NM_000088 & $\begin{array}{l}\text { CACCAATCACCTGCGTACAGAA } \\
\text { CAGATCACGTCATCGCACAAC }\end{array}$ & 118 \\
\hline RPL13a & NM_012423 & $\begin{array}{l}\text { GAGGTATGCTGCCCCACAAA } \\
\text { GTGGGATGCCGTCAAACAC }\end{array}$ & 75 \\
\hline
\end{tabular}

Figure 1. Multipotency of hMSCs.

A. Induction of osteogenic differentiation. After 10 and 20 days of cell culture in osteogenic medium, osteogenic differentiation was assessed by determining the ALP activity and by performing RT-PCR analysis of osterix and osteocalcin expression. ( $\mathrm{n}=1$ donor). $\mathrm{H}_{2} \mathrm{O}$ was used as the negative control for RT-PCR. B. Induction of chondrogenic differentiation. After 20 and 30 days of cell culture in chondrogenic medium, chondrogenic differentiation was assessed by performing immuno-histological analysis of human type II collagen expression. Sections were counter-stained using haematoxylin. Incubation with secondary antibody alone was used as the negative control. Scale bar $=10 \mu \mathrm{m}$. ( $\mathrm{n}=1$ donor). C. Induction of adipogenic differentiation. After 10 and 20 days of cell culture in adipogenic medium, adipogenic differentiation of hMSCs was assessed by Oil Red O staining, and the levels of aP2 and PPAR $\gamma$ expression were determined by performing RT-PCR analysis. $\mathrm{H}_{2} \mathrm{O}$ was used as the negative control for RT-PCR. Scale bar $=50 \mu \mathrm{m}$. $(\mathrm{n}=1 \mathrm{donor})$.

A
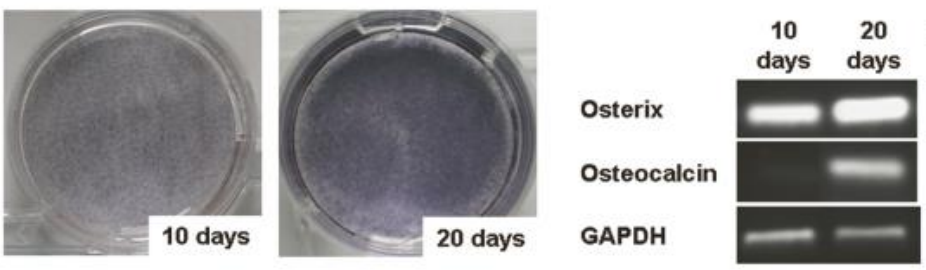

Negative

B
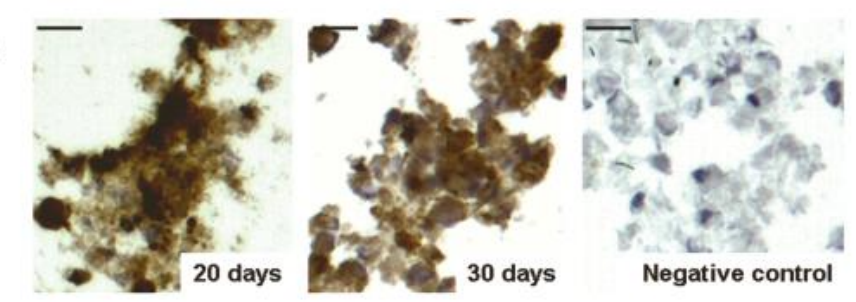

C
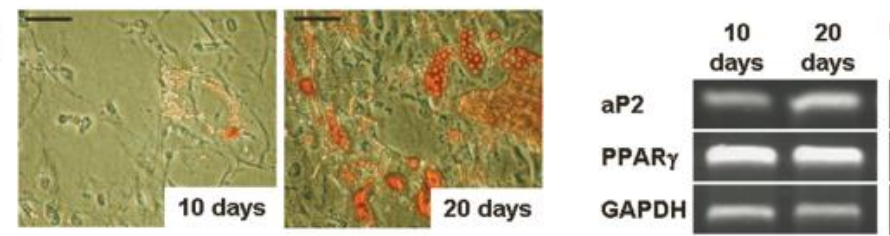

Negative Control

aP

GAPDH Control

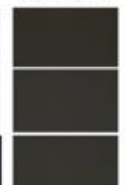

\section{1}

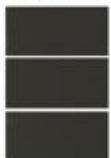


Figure 2. $\mathrm{pO}_{2}$ levels with time in the hypoxic system.

Cell culture medium was placed in a sealed jar containing an oxygen chelator. Twice a day and during 5 days, $\mathrm{pO}_{2}$ levels were measured with a pO $\mathrm{O}_{2}$ oxygen sensor without opening the hypoxic system. Values are means \pm SD; in triplicate.

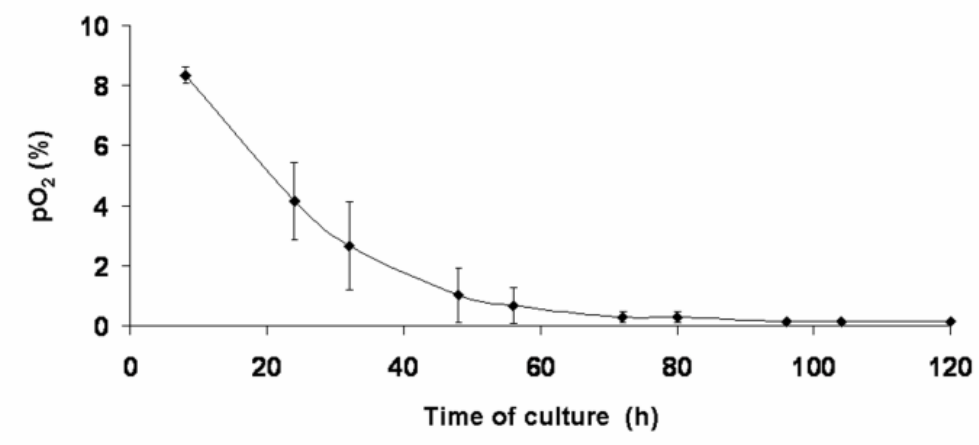

Figure 3. hMSC death rate under hypoxic conditions.

hMSCs were exposed to hypoxic conditions for 48,72 and 120 hours. Cell death rates were assessed by Live/Dead staining followed by image analysis. Values are means $\pm \mathrm{SD} ; \mathrm{n}=3$ donors.

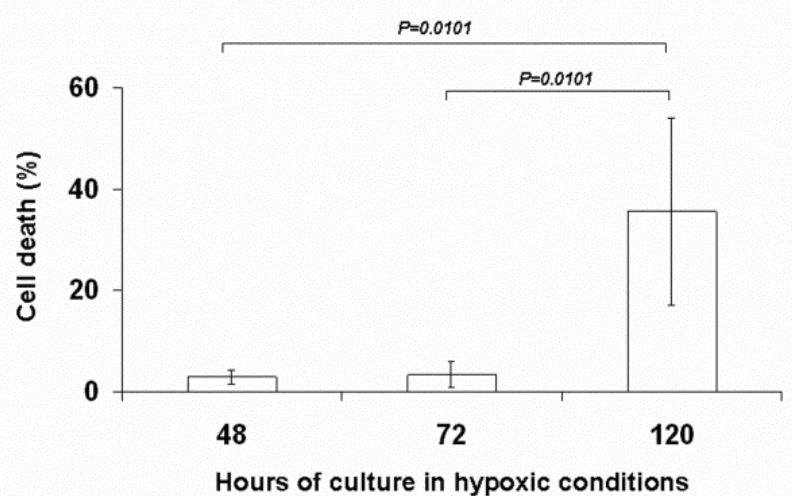


Figure 4. Effects of temporary hypoxia on the osteogenic potential of hMSCs.

hMSCs were exposed to either control $(21 \%$ O2) or hypoxic ( $1 \%$ O2) conditions for 48 hours. After exposure, the media were replaced by osteogenic medium and hMSCs were cultured in control conditions for 0,14 and 28 days. At the end of these time periods, osteoblastic differentiation was evaluated by performing RT-PCR analysis on osteoblastic markers. RPL13a was used as the endogenous reference gene. Results presented here were obtained on one donor representative of the three donors studied.

\section{Days of culture in osteogenic medium}

\begin{tabular}{|c|c|c|c|c|c|c|}
\hline \multirow[b]{2}{*}{ Genes } & \multicolumn{2}{|c|}{0} & \multicolumn{2}{|c|}{14} & \multicolumn{2}{|c|}{28} \\
\hline & $\mathbf{N}$ & H & $\mathbf{N}$ & H & $\mathbf{N}$ & H \\
\hline ALP & $=$ & $=$ & 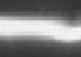 & 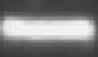 & & \\
\hline BMP2 & 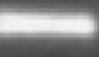 & 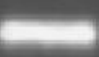 & $=$ & 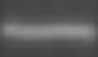 & 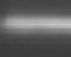 & 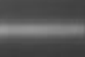 \\
\hline BSP & E & & 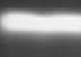 & 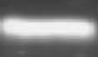 & & \\
\hline Osteopontin & & & & 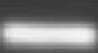 & & 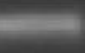 \\
\hline Cbfa 1/Runx2 & $=$ & 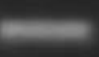 & 2 & 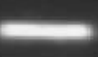 & & - \\
\hline Osteocalcin & 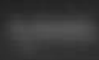 & & $\because$ & n & 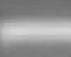 & \\
\hline Type I collag. & 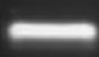 & 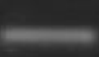 & n & - & 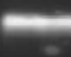 & $=$ \\
\hline RPL13a & - & $=$ & & 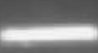 & - & \\
\hline
\end{tabular}




\section{Figure 5. Effects of temporary hypoxia on the cbfa-1/Runx2, osteocalcin and type I collagen expression by hMSCs.}

hMSCs were exposed to either control $\left(21 \% \mathrm{O}_{2}\right.$; white bars) or hypoxic $\left(1 \% \mathrm{O}_{2}\right.$; grey bars) conditions for 48 hours. After exposure, the media were replaced by osteogenic medium and hMSCs were cultured in control conditions for 0, 14 and 28 days. At the end of these time periods, mRNA expression levels of cbfa-1/Runx2, osteocalcin and type I collagen were determined by performing Real-Time PCR. RPL13a was used as the endogenous reference gene. Values are means $\pm \mathrm{SD} ; \mathrm{n}=3$ donors; the assays performed on each donor were carried out in triplicate.
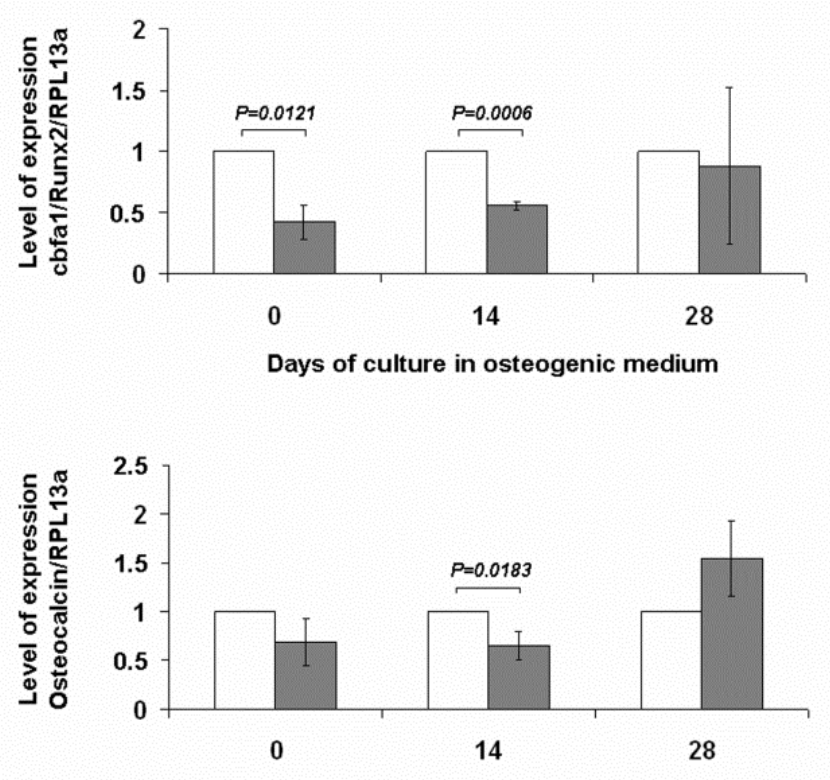

Days of culture in osteogenic medium

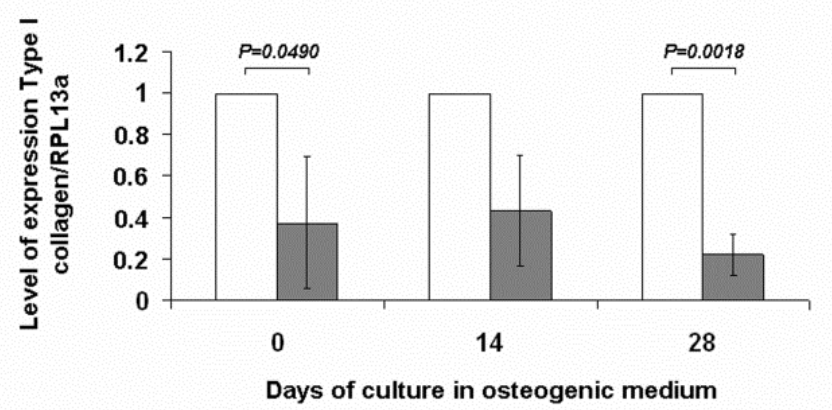


Potier E. et al.

Figure 6. Effects of temporary hypoxia on the mRNA expression of angiogenic factors by hMSCs.

hMSCs were exposed to either control $\left(21 \% \mathrm{O}_{2}\right.$; white bars) or hypoxic $\left(1 \% \mathrm{O}_{2}\right.$; grey bars) conditions for 48 hours. Expression levels of TGF $\beta 1$, TGF $\beta 2$, TGF $\beta 3$, bFGF, and VEGF were normalized using the respective expression levels of RPL13a. Values are means \pm SD; $n=3$ donors.
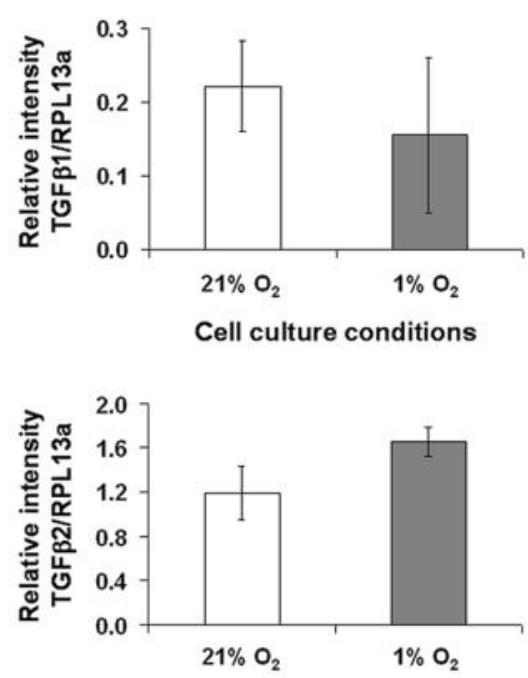

Cell culture conditions
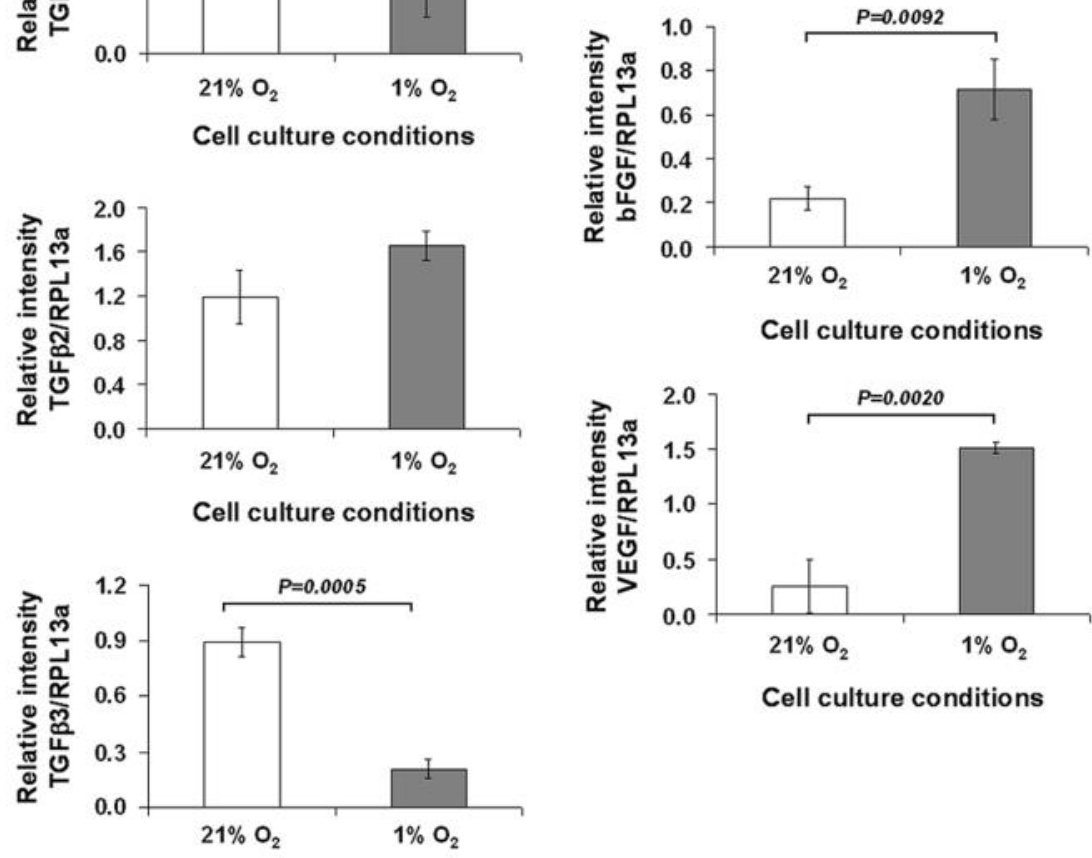

Cell culture conditions

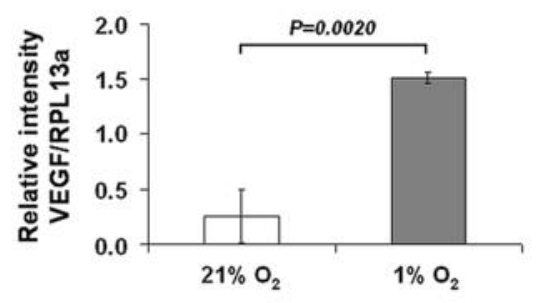

Cell culture conditions

Cell culture conditions 
Figure 7. Effects of temporary hypoxia on the protein secretion of three major regulators of angiogenesis by hMSCs.

hMSCs were exposed to either control $\left(21 \% \mathrm{O}_{2}\right.$; white bars) or hypoxic $\left(1 \% \mathrm{O}_{2}\right.$; grey bars) conditions for 48 hours. The secretion levels of TGF $\beta 1$ (A), bFGF (B) and VEGF (C) were then determined using ELISA assays. Values are means \pm SD; $n=3$ donors.

A.

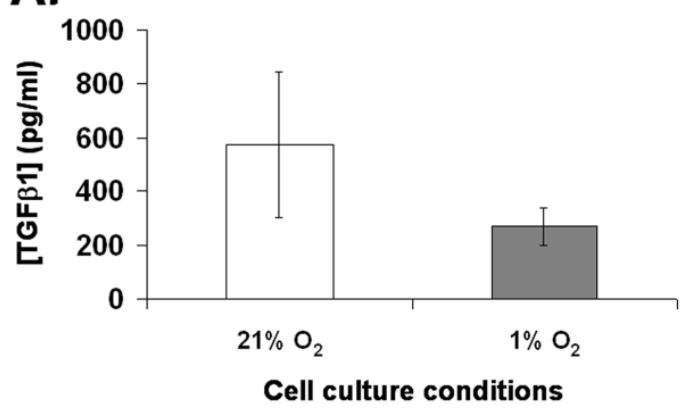

B.
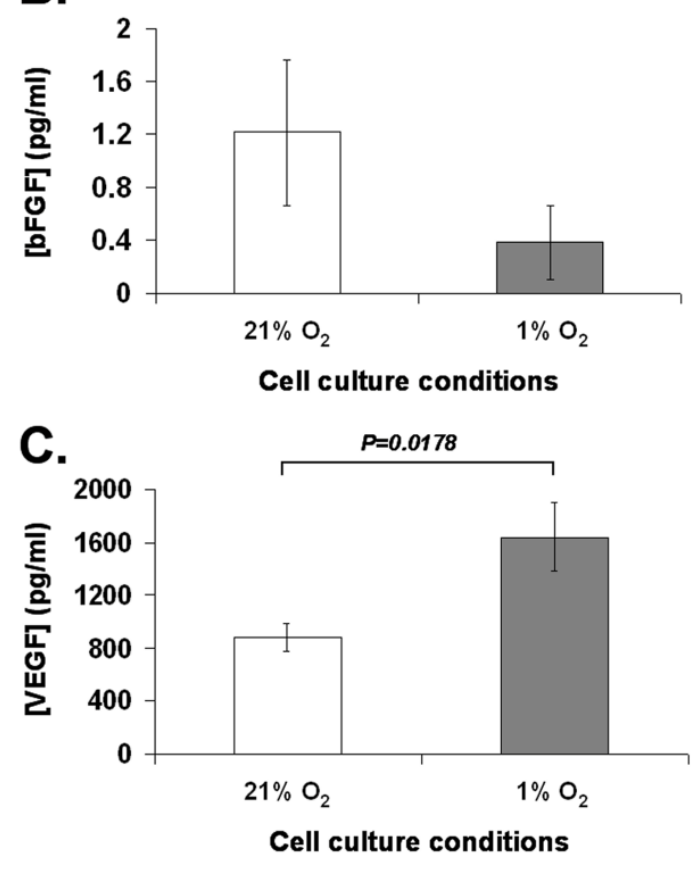